\title{
Tezepelumab Reduces Exacerbations Across All Seasons in Patients with Severe, Uncontrolled Asthma: A Post Hoc Analysis of the PATHWAY Phase 2b Study
}

\author{
This article was published in the following Dove Press journal: \\ Journal of Asthma and Allergy
}

\author{
Jonathan Corren' \\ Martin Karpefors $\mathbb{D}^{2}$ \\ Åsa Hellqvist ${ }^{3}$ \\ Jane R Parnes ${ }^{4}$ \\ Gene Colice ${ }^{5}$ \\ 'Departments of Medicine and Pediatrics, \\ David Geffen School of Medicine, \\ University of California, Los Angeles, CA, \\ USA; '2Data Science and Al, AstraZeneca, \\ Gothenburg, Sweden; ${ }^{3}$ Biometrics, Late- \\ Stage Development, Respiratory and \\ Immunology, AstraZeneca, Gothenburg, \\ Sweden; ${ }^{4}$ Amgen, Thousand Oaks, CA, \\ USA; ${ }^{5}$ Late-Stage Development, \\ Respiratory and Immunology, \\ AstraZeneca, Gaithersburg, MD, USA
}

Introduction: Tezepelumab is a human monoclonal antibody that blocks thymic stromal lymphopoietin (TSLP), an epithelial cytokine implicated in airway inflammation in asthma, from binding to its heterodimeric receptor. In the PATHWAY phase $2 b$ study, tezepelumab significantly reduced exacerbation rates compared with placebo in adults with severe, uncontrolled asthma, irrespective of baseline disease characteristics.

Objective: To evaluate the effect of tezepelumab on asthma exacerbations on a seasonal basis. Methods: This was a post hoc analysis of the PATHWAY study (NCT02054130). Adults $(\mathrm{N}=550)$ with severe, uncontrolled asthma were randomized 1:1:1:1 to receive subcutaneous tezepelumab $70 \mathrm{mg}$ every 4 weeks (Q4W), $210 \mathrm{mg}$ Q4W or $280 \mathrm{mg}$ every 2 weeks (Q2W), or placebo Q2W, for 52 weeks. The annualized asthma exacerbation rate (AAER), total number of days with an exacerbation, proportion of patients with at least one exacerbation or 0,1 or $\geq 2$ exacerbations, and proportion of patients experiencing an exacerbation per day were evaluated by season and over the year, by treatment in the overall study population and in subgroups according to baseline blood eosinophil count ( $\geq 300$ cells $/ \mu \mathrm{L}$ or $<300$ cells $/ \mu \mathrm{L}$ ) or atopic asthma status (fluoro-enzyme immunoassay [FEIA]+ or FEIA-).

Results: Seasonal variations in exacerbation rates were found, with peaks observed in fall and winter, and greater variations in patients with high blood eosinophil counts ( $\geq 300$ cells/ $\mu \mathrm{L})$. Tezepelumab treatment consistently reduced exacerbation rates across all seasons compared with placebo. Furthermore, there was a trend, which was not significant, toward a reduction in the total number of days with exacerbations and in the proportion of patients with exacerbations during each season in patients treated with tezepelumab compared with those who received placebo, irrespective of blood eosinophil count or atopic asthma status. Conclusion: Tezepelumab reduced exacerbations across all seasons, irrespective of evaluated baseline disease characteristics. These data support the efficacy of tezepelumab in a broad population of patients with severe, uncontrolled asthma.

Keywords: allergens, asthma, eosinophil, seasonal variation, tezepelumab, thymic stromal lymphopoietin

\section{Introduction}

Asthma occurs in approximately 339 million individuals globally, ${ }^{1}$ up to $10 \%$ of whom have severe asthma. ${ }^{2}$ A substantial number of patients with severe asthma continue to experience exacerbations involving acute worsening of symptoms despite treatment with medium- to high-dose inhaled corticosteroids (ICS) and
Departments of Medicine and Pediatrics, David Geffen School of Medicine at UCLA, 10833 Le Conte Ave, Los Angeles, CA 90095, USA

Tel + I-3I0-3I2-5050

Email jcorren@ucla.edu
Journal of Asthma and Allergy 202I:14 I-II

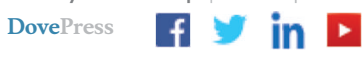

http://doi.org/10.2147/IAA. 2826036 
long-acting $\beta_{2}$-agonists (LABAs), as well as additional controller therapies. ${ }^{3-5}$ These patients have a high burden of disease and are at risk for recurrent exacerbations and hospital admissions. ${ }^{6-8}$

Seasonal variations in asthma exacerbations have been observed in both epidemiological and clinical studies, with higher rates of exacerbations in adults usually occurring in fall and winter. ${ }^{9-12}$ Seasonal environmental changes in both aeroallergen exposure and the prevalence of respiratory viral infections, including those caused by influenza viruses and, in particular, rhinoviruses, have been implicated in the amplification of airway inflammation in patients with asthma. ${ }^{13,14}$ This increased inflammation may induce asthma exacerbations and acute worsening of symptoms. ${ }^{14}$ Data from a recent study suggest that patients with high blood eosinophil counts ( $\geq 300$ cells $/ \mu \mathrm{L}$ ) appear to experience greater seasonal variability in asthma exacerbations than those with low blood eosinophil counts $(<300$ cells $/ \mu \mathrm{L}){ }^{12}$

Currently approved biological therapies for severe asthma, which are indicated for patients with severe, allergic asthma (monoclonal antibodies directed against immunoglobulin E [IgE; omalizumab]) or severe, eosinophilic asthma (anti-interleukin [IL]-5 [mepolizumab, reslizumab], anti-IL-5 receptor-alpha [benralizumab] and anti-IL -4 receptor-alpha [dupilumab]), reduce asthma exacerbation rates in study populations by approximately $50 \% .{ }^{15-17}$ Omalizumab has been shown to blunt seasonal exacerbations more effectively in the fall and spring seasons than in summer, ${ }^{18}$ whereas mepolizumab and benralizumab have more uniform effects on exacerbations across the seasons. ${ }^{11,19}$

Tezepelumab is a human monoclonal antibody (IgG2 $\lambda$ ) that binds specifically to thymic stromal lymphopoietin (TSLP), blocking it from interacting with its heterodimeric receptor. $^{20,21}$ TSLP is an upstream epithelial cytokine released in response to airborne triggers of asthma, including allergens, viruses, cigarette smoke and other pollutants interacting with the airway epithelium, and is implicated in the initiation and persistence of airway inflammation in asthma. $^{22-24}$ In the PATHWAY phase $2 b$ study (ClinicalTrials.gov identifier: NCT02054130), tezepelumab significantly reduced the annualized asthma exacerbation rate (AAER) by up to $71 \%$ compared with placebo in adults with severe, uncontrolled asthma. ${ }^{21}$ Reductions in exacerbations were observed irrespective of baseline disease characteristics, suggestive of a potential benefit of tezepelumab treatment in a broad population of patients relative to other approved biological therapies. ${ }^{21}$ This post hoc analysis evaluated the effect of tezepelumab treatment on asthma exacerbations in the PATHWAY study population on a seasonal basis.

\section{Methods}

\section{Study Design and Participants}

PATHWAY was a phase $2 \mathrm{~b}$, randomized, multicenter, double-blind, placebo-controlled trial, conducted between December 2013 and March 2017. The full design and inclusion and exclusion criteria of this study have been described previously. ${ }^{21}$ Patients included in the study were 18-75 years old and were current non-smokers with severe asthma that was uncontrolled, despite treatment with medium- or high-dose ICS (250-500 $\mu \mathrm{g} /$ day or $>500 \mu \mathrm{g} /$ day of fluticasone dry powder inhaler or equivalent, respectively) plus a LABA. Patients were required to have a history of at least two asthma exacerbations that led to systemic corticosteroid treatment, or at least one severe exacerbation that resulted in hospitalization, in the 12 months before study entry.

Patients with any clinically important pulmonary disease other than asthma were excluded. Randomization was stratified according to study site (Japanese or nonJapanese) and subsequently by blood eosinophil count $(\geq 250$ cells $/ \mu \mathrm{L}$ or $<250$ cells $/ \mu \mathrm{L})$ and ICS dose level (medium or high). Patients were randomized (1:1:1:1) to receive subcutaneous doses of tezepelumab $70 \mathrm{mg}$ every 4 weeks (Q4W), $210 \mathrm{mg}$ Q4W or $280 \mathrm{mg}$ every 2 weeks (Q2W), or placebo Q2W, for 52 weeks.

The study was conducted in accordance with the ethical principles of the Declaration of Helsinki, International Conference for Harmonisation Good Clinical Practice guidelines, and applicable regulatory requirements. Approvals from Copernicus central Institutional Review Board (Durham, NC, USA) and local independent ethics committees were obtained, and all patients provided written informed consent in accordance with local requirements. A full list of the Institutional Review Boards and independent ethics committees is provided in Table S1.

\section{Outcomes}

The primary outcome measured in this analysis was the AAER, which was evaluated by season for each treatment group. An asthma exacerbation was defined as worsening of asthma that led to use of systemic corticosteroids for at 
least 3 days, an emergency room visit that required systemic corticosteroids for at least 3 days, or hospitalization.

In addition, the total number of days with an exacerbation per patient, the proportion of patients with at least one exacerbation or 0,1 or $\geq 2$ exacerbations, and the proportion of patients experiencing an exacerbation per day were evaluated by season and over the year; these outcomes were assessed by treatment in the overall study population and in subgroups according to central blood eosinophil count ( $\geq 300$ cells $/ \mu \mathrm{L}$ or $<300$ cells $/ \mu \mathrm{L})$ or atopic asthma status (fluoro-enzyme immunoassay [FEIA]+ or FEIA - for IgE-mediated asthma) at baseline (defined as the last valid assessment before the first administration of study drug on day 1). The allergens tested for in the specific IgE FEIA are listed in Table S2.

Data presented will focus primarily on the tezepelumab $210 \mathrm{mg}$ (selected for phase 3 clinical studies) and pooled tezepelumab dose groups.

\section{Statistical Analyses}

Exacerbations were assigned to a single season in which the exacerbation began. For exacerbations that extended over several seasons, the number of days of the exacerbation that fell within a defined seasonal period contributed to that period accordingly. Winter, spring, summer and fall were defined as January to March, April to June, July to September and October to December, inclusive, respectively. Data from patients in the southern hemisphere were transformed to align with equivalent seasonal time periods in the northern hemisphere by shifting the data by 6 months (ie, July would become month 1 and January would become month 7). To visualize the annual exacerbation pattern, each exacerbation was mapped onto a day of a 365-day year. Given that 2016 was a leap year, all data from February 29, 2016 to December 31, 2016 were assigned to the previous day; to keep exacerbation episodes spanning February 29, 2016 the correct length, an extra day was added to the end of these episodes. Area plots were generated to show, on a day-by-day basis, the sum of patients experiencing an exacerbation divided by the number of patients. Area plots were also generated for each subgroup where appropriate.

AAERs and 95\% confidence intervals (CIs) during each season were estimated using a negative binomial regression model, with treatment group (placebo or tezepelumab) and baseline ICS dose level (medium or high) as covariates. The logarithm of the time at risk within each season was included as an offset in the model. Owing to convergence issues, the baseline blood eosinophil count stratification factor $(\geq 250$ cells $/ \mu \mathrm{L}$ or $<250$ cells $/ \mu \mathrm{L})$ was not included as a covariate in the model.

Descriptive summary statistics (n [\%] or mean [standard deviation; SD]) were used to report: total number of exacerbations per patient during each season; total number of days with an exacerbation per patient during each season and weekly; proportion of patients with at least one exacerbation or 0,1 or $\geq 2$ exacerbations during each season; and proportion of patients experiencing an exacerbation per day throughout the year (for the overall population, and for subgroups of patients with high $[\geq 300$ cells/ $\mu \mathrm{L}]$ or low $[<300$ cells $/ \mu \mathrm{L}]$ baseline blood eosinophil counts and with FEIA+ or FEIA- atopic status).

As an additional exploratory analysis, the relationship between exacerbations and the influenza season was visualized graphically by plotting the proportion of Eastern European patients experiencing an exacerbation per day together with the mean proportion of positive influenza tests among respiratory specimens in Eastern Europe (after aligning at the median peak for the period comprising the 2003-2004 to 2013-2014 seasons, excluding 2009-2010). ${ }^{25}$ Eastern Europe, consisting of Bulgaria, Czech Republic, Hungary, Latvia, Lithuania, Serbia, Slovakia and Ukraine, was selected for this analysis because patients from these countries comprised the largest regional population of the PATHWAY study.

All analyses were conducted without control of type 1 error; as such, $p$ values are not presented.

\section{Results}

\section{Patient Disposition}

Overall, 550 patients were randomized to receive tezepelumab $70 \mathrm{mg}$ Q4W (n=138), $210 \mathrm{mg}$ Q4W $(\mathrm{n}=137)$ or $280 \mathrm{mg}$ Q2W ( $\mathrm{n}=137)$, or placebo Q2W ( $=138)$. Patient demographics and clinical characteristics at baseline have been reported previously. ${ }^{21}$

The overall proportion of patients in the combined placebo and pooled tezepelumab groups with a history of at least three exacerbations at baseline (ie, in the 12 months before study start) was slightly higher in patients with high ( $\geq 300$ cells $/ \mu \mathrm{L})$ vs low $(<300$ cells $/ \mu \mathrm{L})$ baseline blood eosinophil counts $(22.8 \%$ vs $17.1 \%$, respectively), and in patients with non-atopic asthma (FEIA-) versus atopic asthma (FEIA +$)(21.1 \%$ vs $18.5 \%$, respectively; Table S3). 


\section{Effect of Tezepelumab Treatment on Exacerbation Frequency by Season}

Overall, across treatment groups, AAER was highest in winter, followed by fall and summer, and lowest in spring. Treatment with tezepelumab $210 \mathrm{mg}$ Q4W reduced AAER by $64 \%$ (95\% CI 22 to 83 ), $80 \%$ (95\% CI 41 to 93 ), $82 \%$ (95\% CI 48 to 94 ) and 67\% (95\% CI 21 to 86), compared with placebo, in winter, spring, summer and fall, respectively (Figure 1; Table S4). For the pooled tezepelumab group, AAER was reduced by $63 \%$ (95\% CI 37 to 78 ), $70 \%$ (95\% CI 42 to 85 ), $77 \%$ (95\% CI 56 to 88 ) and $63 \%$ (95\% CI 34 to 79 ), compared with placebo, in winter, spring, summer and fall, respectively.

\section{Number of Days with Exacerbations During Each Season and Throughout the Year}

Patients treated with tezepelumab experienced fewer days with exacerbations than those who received placebo. In the placebo group, the total number of days with an exacerbation were 336, 217, 255 and 323 during winter, spring, summer and fall, respectively, compared with 81, 46, 31 and 89 days in the tezepelumab $210 \mathrm{mg}$ Q4W group, respectively, and 353, 189, 203 and 304 days in the pooled tezepelumab group, respectively. During each season, the mean $[\mathrm{SD}]$ number of days with an exacerbation per patient in the placebo group was higher (2.5 [7.2], 1.6 [4.9], 1.9 [5.5] and 2.3 [7.4] days for winter, spring, summer and fall, respectively) than in the tezepelumab $210 \mathrm{mg}$ Q4W group (0.6 [2.8], 0.4 [2.0], 0.2 [1.6] and 0.7 [3.2] days, respectively) and the pooled tezepelumab group (0.9 [3.7], 0.5 [2.8], 0.5 [3.0] and 0.8 [3.3] days, respectively) (Figure 2). These observations were also true for both the high and low blood eosinophil count subgroups, and the atopic and nonatopic asthma subgroups (Tables S5 and S6). In general, irrespective of treatment, patients with high blood eosinophil counts experienced more days with exacerbations than those with low blood eosinophil counts. Patients with both atopic and non-atopic asthma experienced more days with exacerbations during winter than in the other seasons, while patients with atopic asthma also experienced more days with exacerbations in fall.

When presented by week, the mean number of days with an exacerbation per patient was generally lower for the tezepelumab $210 \mathrm{mg}$ Q4W and pooled dose groups than for the placebo group throughout the year (Figure 3).

\section{Proportion of Patients with Exacerbations During Each Season and} Throughout the Year

The proportion of patients who experienced at least one exacerbation was higher in all seasons in the placebo

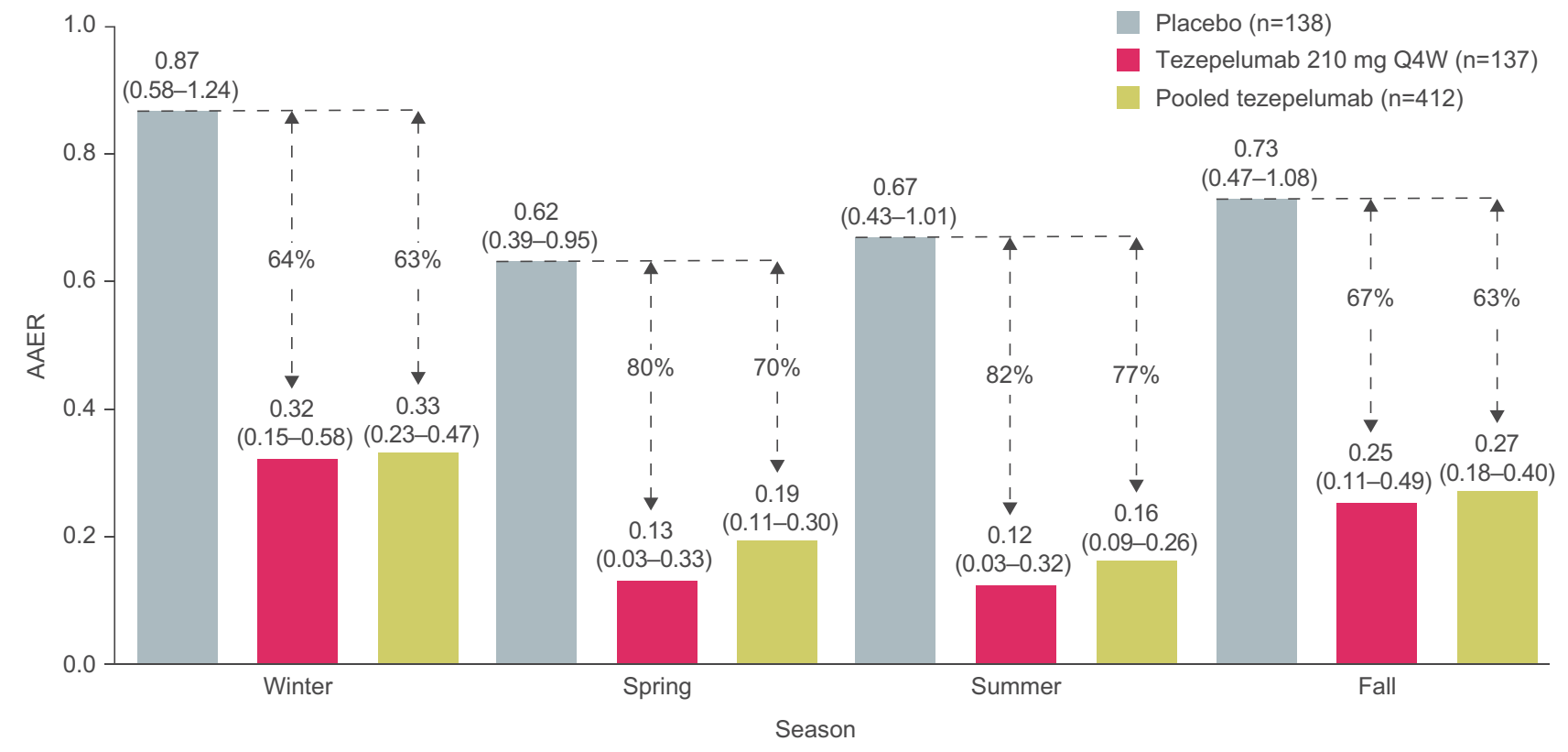

Figure I AAER during each season.

Note: Data are annualized rates and $95 \%$ Cls.

Abbreviations: $\mathrm{AAER}$, annualized asthma exacerbation rate; $\mathrm{Cl}$, confidence interval; $\mathrm{Q} 4 \mathrm{~W}$, every 4 weeks. 


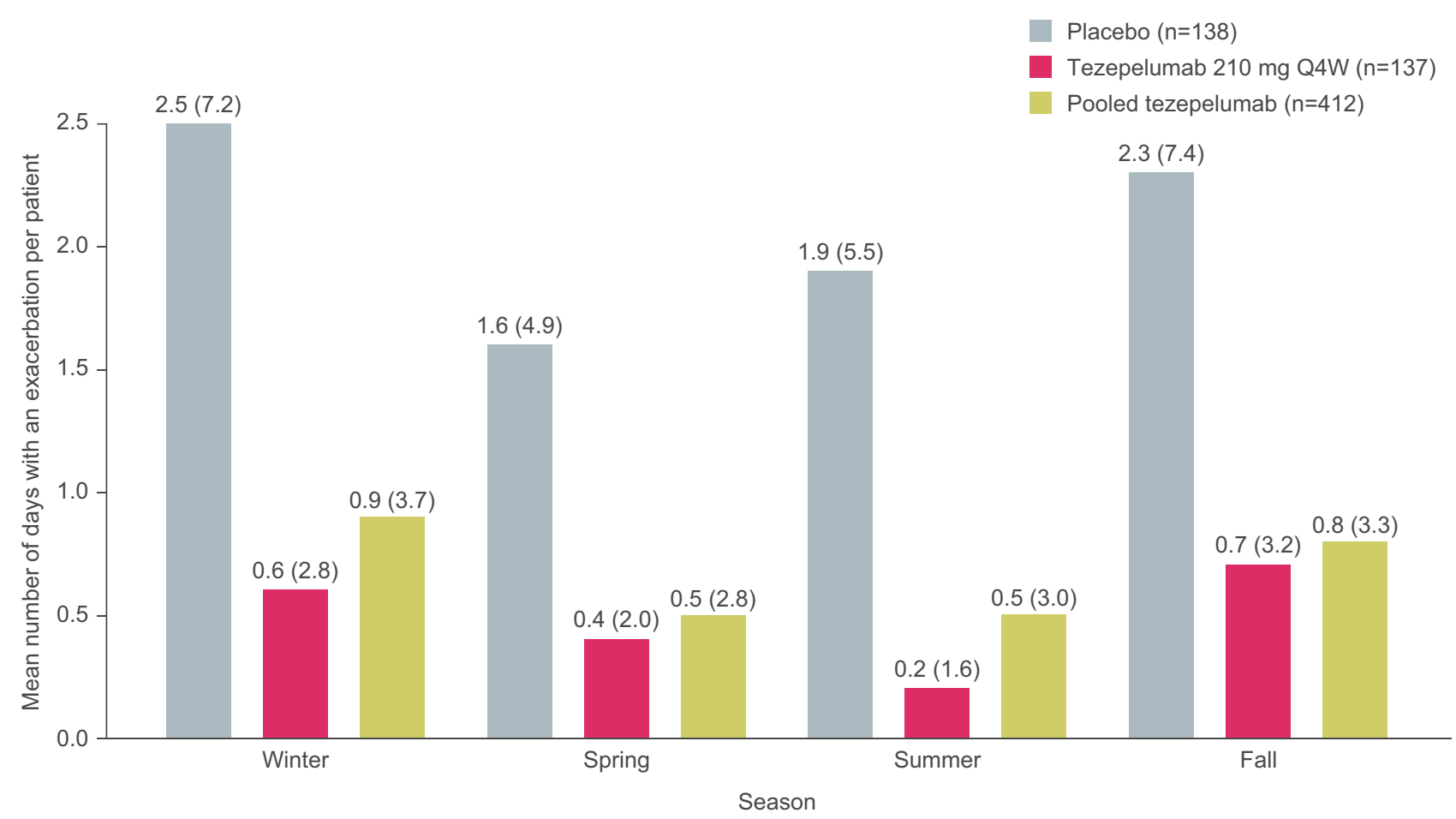

Figure 2 Mean number of days with an asthma exacerbation per patient, by season.

Notes: Standard deviations are provided in parentheses. Winter, spring, summer and fall are defined as Jan-Mar, Apr-Jun, Jul-Sep and Oct-Dec, respectively.

group $(16.9 \%, 13.1 \%, 15.4 \%$ and $14.5 \%$ during winter, spring, summer and fall, respectively) than in the tezepelumab $210 \mathrm{mg}$ Q4W group (6.9\%, 3.1\%, 3.1\% and $5.3 \%$, respectively) and the pooled tezepelumab group $(7.9 \%, 4.1 \%, 4.0 \%$ and $6.4 \%$, respectively) (Table 1). This finding was also observed in both the high and low blood eosinophil count subgroups (Table S5) and the atopic and non-atopic subgroups (Table S6). Correspondingly, there were more patients with no exacerbations in the tezepelumab-treated subgroups with high and low blood eosinophils and atopic and non-atopic status, during each season, than in the subgroups who received placebo (Tables $\mathrm{S} 5$ and $\underline{\mathrm{S} 6}$ ). In general, irrespective of treatment, patients with both atopic and non-atopic asthma experienced more exacerbations during winter than in the other seasons, and patients with atopic asthma also experienced a peak in exacerbations in fall.

Peaks in the proportion of patients with exacerbations per day were observed in winter, early summer, and fall (Figure 4). The proportion of patients with exacerbations per day was generally lower in the tezepelumab $210 \mathrm{mg}$ and pooled tezepelumab dose groups than in the placebo group throughout the year, which was also the case in both blood eosinophil count

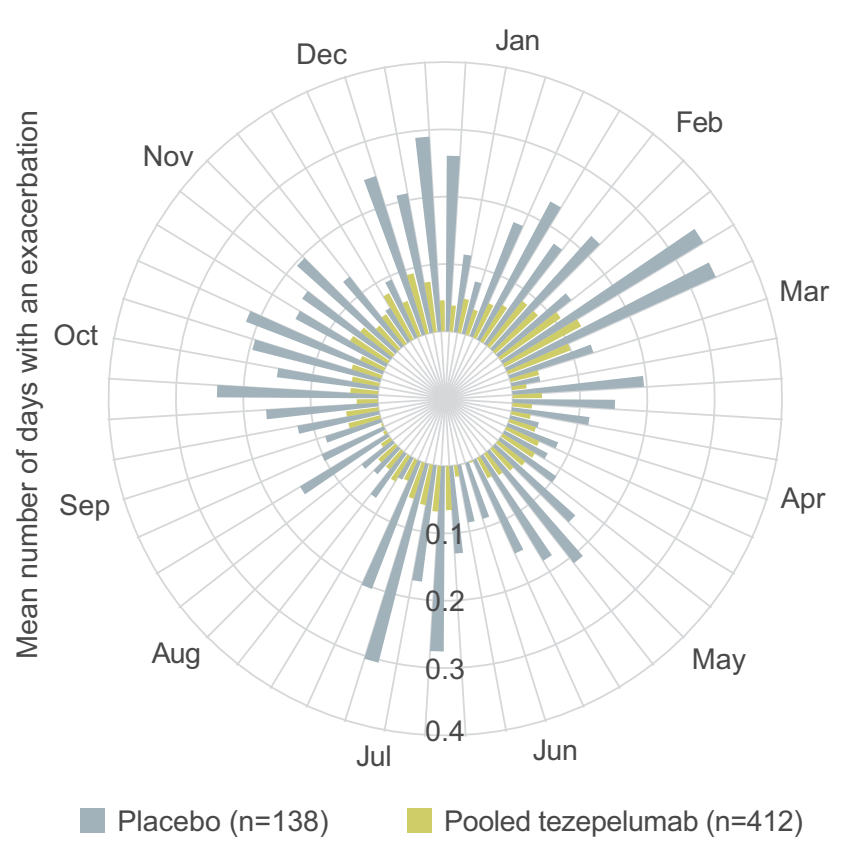

Figure 3 Mean number of days with an asthma exacerbation per patient, for each week of the year.

Notes: Weeks are defined as 7-day periods starting on January I; day 365 was added to week 52, resulting in an 8-day week.

subgroups and both atopic asthma status subgroups (Figure 5). The greatest peak in the proportion of Eastern European patients with exacerbations per day 
Table I Number of Patients with Asthma Exacerbations by Season

\begin{tabular}{|c|c|c|c|}
\hline & $\begin{array}{l}\text { Placebo } \\
(n=138)\end{array}$ & $\begin{array}{l}\text { Tezepelumab } \\
210 \mathrm{mg} \text { Q4W } \\
(\mathrm{n}=137)\end{array}$ & $\begin{array}{l}\text { Pooled } \\
\text { Tezepelumab } \\
(n=4 \mid 2)\end{array}$ \\
\hline \multicolumn{4}{|c|}{$\begin{array}{l}\text { Number of patients with at least one exacerbation during season } \\
n(\%)\end{array}$} \\
\hline Winter & $\begin{array}{l}23(16.9) \\
(n=136)\end{array}$ & $\begin{array}{l}9(6.9) \\
(n=130)\end{array}$ & $\begin{array}{l}31(7.9) \\
(n=394)\end{array}$ \\
\hline Spring & $\begin{array}{l}18(13.1) \\
(n=137)\end{array}$ & $\begin{array}{l}4(3.1) \\
(n=129)\end{array}$ & $\begin{array}{l}16(4.1) \\
(n=395)\end{array}$ \\
\hline Summer & $\begin{array}{l}21(15.4) \\
(n=136)\end{array}$ & $\begin{array}{l}4(3.1) \\
(n=131)\end{array}$ & $\begin{array}{l}16(4.0) \\
(n=403)\end{array}$ \\
\hline Fall & $\begin{array}{l}20(14.5) \\
(n=138)\end{array}$ & $\begin{array}{l}7(5.3) \\
(n=133)\end{array}$ & $\begin{array}{l}26(6.4) \\
(n=404)\end{array}$ \\
\hline \multicolumn{4}{|c|}{$\begin{array}{l}\text { Number of patients with } 0,1 \text { or } \geq 2 \text { exacerbations during season, } \\
n(\%)\end{array}$} \\
\hline \multicolumn{4}{|l|}{ Winter } \\
\hline$n$ & 136 & 130 & 394 \\
\hline 0 & $113(83.1)$ & $|2|(93.1)$ & $363(92.1)$ \\
\hline 1 & $18(13.2)$ & $8(6.2)$ & $30(7.6)$ \\
\hline$\geq 2$ & $5(3.7)$ & I (0.8) & I $(0.3)$ \\
\hline \multicolumn{4}{|l|}{ Spring } \\
\hline$n$ & 137 & 129 & 395 \\
\hline 0 & $119(86.9)$ & $125(96.9)$ & 379 (95.9) \\
\hline 1 & $15(10.9)$ & $4(3.1)$ & $14(3.5)$ \\
\hline$\geq 2$ & $3(2.2)$ & $0(0.0)$ & $2(0.5)$ \\
\hline \multicolumn{4}{|l|}{ Summer } \\
\hline$n$ & 136 & $13 \mid$ & 403 \\
\hline 0 & II (84.6) & 127 (96.9) & $387(96.0)$ \\
\hline 1 & $19(14.0)$ & $4(3.1)$ & $16(4.0)$ \\
\hline$\geq 2$ & $2(1.5)$ & $0(0.0)$ & $0(0.0)$ \\
\hline \multicolumn{4}{|l|}{ Fall } \\
\hline$n$ & 138 & 133 & 404 \\
\hline 0 & II 8 (85.5) & $126(94.7)$ & $378(93.6)$ \\
\hline I & $16(11.6)$ & $6(4.5)$ & $25(6.2)$ \\
\hline$\geq 2$ & $4(2.9)$ & I $(0.8)$ & I $(0.2)$ \\
\hline
\end{tabular}

Note: Data presented are descriptive and no formal statistical analyses were performed.

Abbreviation: Q4W, every 4 weeks.

occurred during winter, which coincided with the annual peak in the proportion of positive influenza tests recorded in that region (Figure 6). On average, fewer patients treated with tezepelumab had exacerbations during the peak of positive influenza tests than those who received placebo.

\section{Discussion}

In the PATHWAY phase $2 \mathrm{~b}$ study, tezepelumab significantly reduced the AAER compared with placebo in adults with severe, uncontrolled asthma. ${ }^{21}$ This post hoc analysis extended this observation by demonstrating that tezepelumab reduced the AAER compared with placebo across all seasons, irrespective of baseline blood eosinophil count and specific IgE (FEIA) status.

Seasonal variations in exacerbation rate (encompassing both frequency and duration) were observed in this study population, with peaks occurring in fall and winter. This is consistent with findings from previous studies of seasonal variation in asthma exacerbations. ${ }^{9-12}$ Interestingly, a somewhat smaller peak in exacerbation rates was also observed during summer in our data set. The greatest peak in the daily proportion of Eastern European patients experiencing an exacerbation came in winter and coincided with the peak of the regional influenza season. Seasonal variation in exacerbations was observed in both patients with high $(\geq 300$ cells $/ \mu \mathrm{L})$ and low $(<300$ cells $/ \mu \mathrm{L})$ baseline blood eosinophil counts, although there appeared to be more exacerbations and also greater seasonal variability in the former group among placebo-treated patients. Similar observations were made in placebo-treated patients with moderate-to-severe asthma in a study of the anti-IL -13 monoclonal antibody lebrikizumab, which used the same blood eosinophil count threshold. ${ }^{12}$ We also observed that patients with non-atopic asthma (FEIA-) experienced the greatest peak in exacerbations in winter, whereas those with atopic asthma (FEIA +$)$ experienced the greatest peaks during both fall and winter.

Treatment with tezepelumab consistently reduced exacerbations throughout the seasons; the AAER was reduced by $64-82 \%$ with tezepelumab $210 \mathrm{mg}$ Q4W compared with placebo, with the greatest reductions observed during summer and the smallest reductions observed during winter. In addition, treatment with tezepelumab reduced both the number of exacerbations during each season and the total days of exacerbations during each season, compared with placebo. These data indicate that asthma exacerbations in the group of patients treated with tezepelumab were less frequent and shorter in length.

Previously, in PATHWAY, tezepelumab treatment was shown to significantly improve the AAER in patients with either high or low blood eosinophil counts, and with either atopic or non-atopic asthma (as determined by FEIA status), 


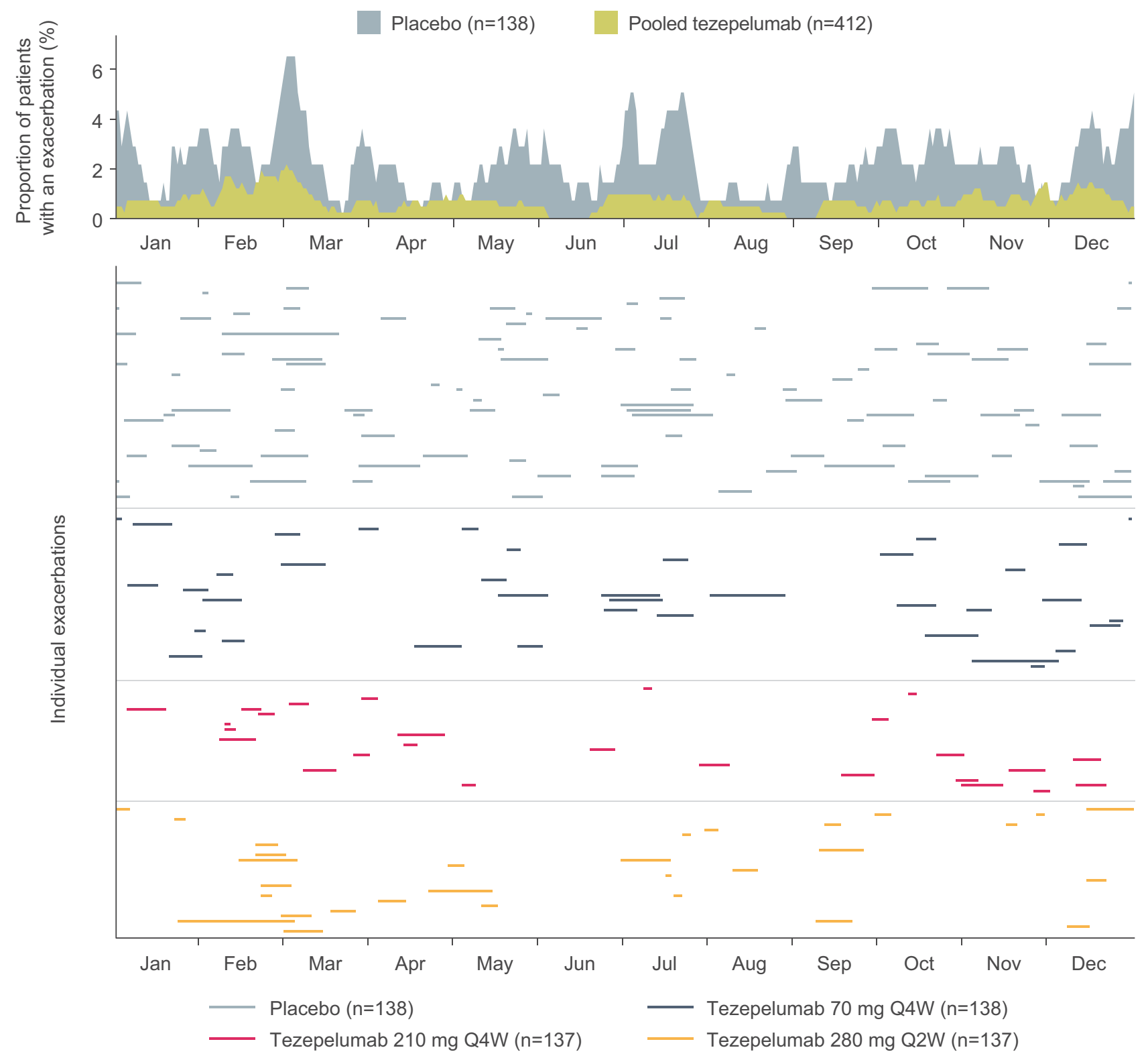

Figure 4 Proportion of patients with asthma exacerbations per day throughout the year.

Notes: The upper part of the figure shows the marginal overlapping area plot of exacerbations per day throughout the year, expressed as percentage of placebo patients and percentage of all tezepelumab treatment arms summed. The lower part of the figure shows the occurrence and duration of individual exacerbations. Treatment arms are grouped between horizontal dividers.

Abbreviations: Q2W, every 2 weeks; Q4W, every 4 weeks.

at baseline. ${ }^{21}$ In the present analysis, tezepelumab was again shown to reduce asthma exacerbations in all baseline blood eosinophil and atopic asthma subgroups, with a trend, which was not significant, towards reductions in both the number and duration of exacerbations during all seasons, compared with placebo. Studies of other biological therapies in patients with severe asthma have reported reductions in exacerbations during each season; however, these analyses either showed reduced efficacy in patients with low baseline eosinophil counts $(<300 \text { cells } / \mu \mathrm{L})^{12}$ or were only investigated in patients with high baseline eosinophil counts $(\geq 300$ cells $/ \mu \mathrm{L}){ }^{11}$ The ability of tezepelumab to reduce exacerbations in patients irrespective of baseline disease characteristics, including blood eosinophil count and atopic status, and across all seasons suggests that it may have efficacy in a broad population of patients with severe, uncontrolled asthma across the spectrum of exacerbation etiologies. The effect of tezepelumab on seasonal asthma exacerbations is consistent with its mechanism of action, targeting the epithelial cytokine TSLP, which activates 


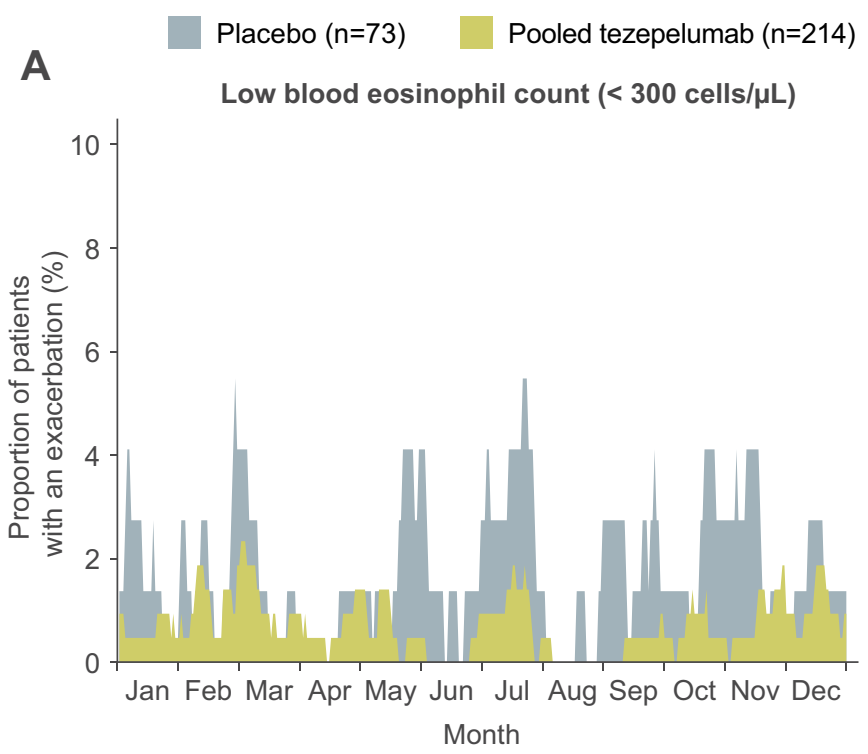

B

Placebo $(n=80) \quad$ Pooled tezepelumab $(n=216)$

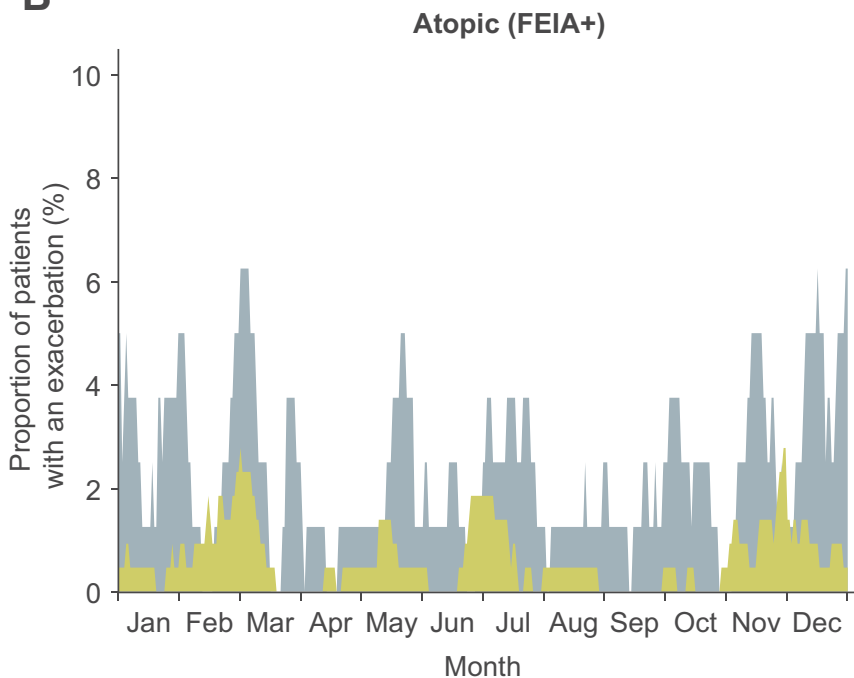

Placebo $(n=65)$

Pooled tezepelumab $(n=198)$

High blood eosinophil count ( $\geq 300$ cells $/ \mu L$ )

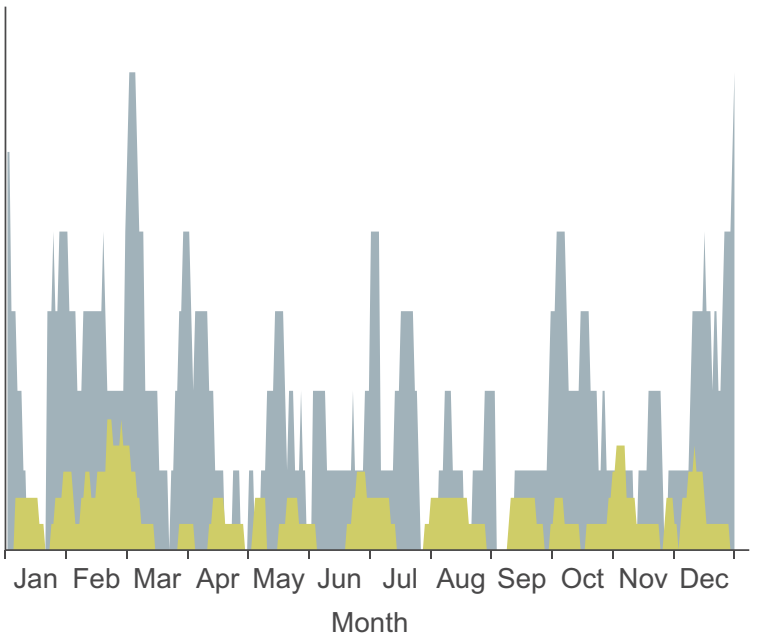

Placebo $(n=50)$

Pooled tezepelumab $(n=168)$

Non-atopic (FEIA-)

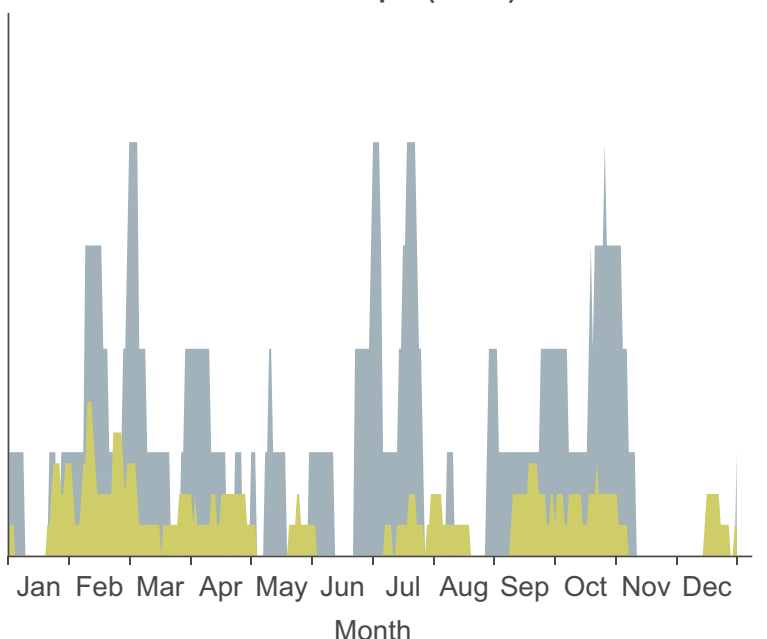

Figure 5 Proportion of patients with asthma exacerbations per day throughout the year, stratified by (A) low ( $<300$ cells $/ \mu \mathrm{L}$; left) or high ( $\geq 300$ cells $/ \mu \mathrm{L}$; right) baseline blood eosinophil counts, or (B) atopic (FEIA+; left) or non-atopic (FEIA-; right) asthma status.

Notes: A number of patients had no atopic status recorded (placebo, $n=8$; pooled tezepelumab, $n=28$ ).

Abbreviations: FEIA, fluoro-enzyme immunoassay; Q4W, every 4 weeks.

downstream inflammatory pathways in response to airborne particles, such as allergens and viruses, interacting with the airway epithelium.

This study has both strengths and weaknesses. An asset of this study is that it included patients with high and low blood eosinophil counts, which allowed insights into the treatment effects of tezepelumab in these two important subgroups. Other valuable aspects of the study design were the duration and regional diversity. The study lasted an entire year, which allowed assessment through multiple viral and allergen seasons, and it was performed in a large number of countries around the world, making the results more generalizable. A limitation of this study was that rigorous assessments of the causes of exacerbations, such as identification of specific viral infections, were not performed. Complete evaluations of atopic status, such as the use of skin testing, were also not performed. In addition, it was not possible to examine the effect of environmental irritants such as smoke and pollution. Owing to the relatively small size of the PATHWAY study population and to the low rate of exacerbations observed in the study, statistical comparisons within seasons across eosinophil and atopic subgroups were not possible, hence these results are 


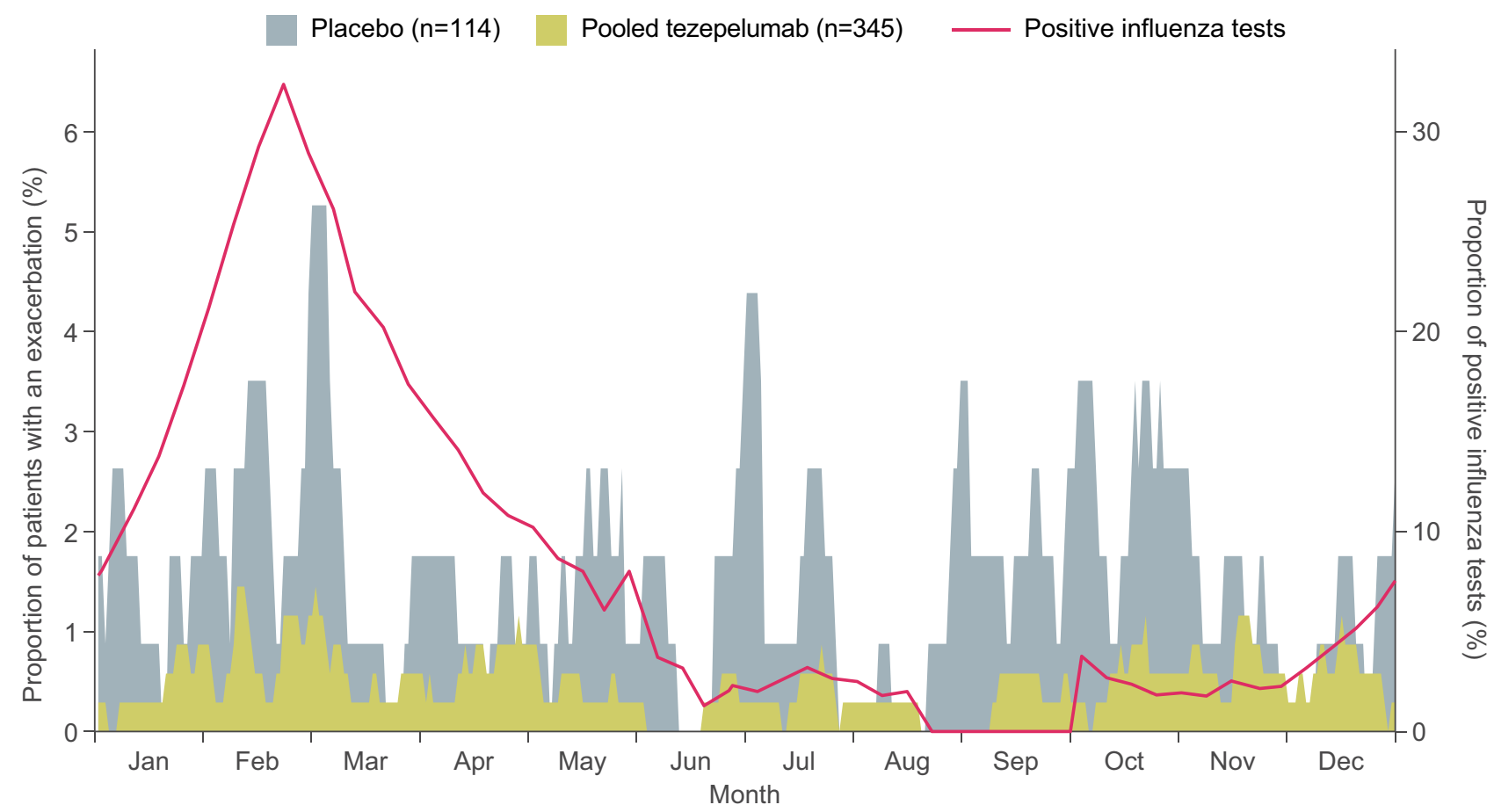

Figure 6 Relationship between asthma exacerbations and the influenza season in Eastern Europe.

Notes: The overlapping area plot shows the proportion of patients with asthma exacerbations per day throughout the year in a subset of patients from Eastern Europe. The purple line, associated with the secondary axis to the right, shows the mean proportion of positive influenza tests for Eastern Europe after aligning at the median peak for the period comprising the 2003-2004 to 2013-2014 seasons (excluding 2009-2010). ${ }^{25}$

presented descriptively only. The effect of tezepelumab across the seasons in these subgroups, and in all patients with severe asthma, will be further assessed in the phase 3 NAVIGATOR study (ClinicalTrials.gov identifier: NCT03347279), which has a considerably larger patient population than PATHWAY.

\section{Conclusions}

In conclusion, treatment with tezepelumab reduced the rate of exacerbations compared with placebo in patients with severe, uncontrolled asthma across all seasons. Exacerbations were reduced irrespective of baseline blood eosinophil count and atopic asthma (FEIA) status. These data support the efficacy of tezepelumab in reducing exacerbations in a broad population of patients with severe, uncontrolled asthma across the spectrum of exacerbation etiologies.

\section{Abbreviations}

AAER, annualized asthma exacerbation rate; CI, confidence interval; FEIA, fluoro-enzyme immunoassay; ICS, inhaled corticosteroids; Ig, immunoglobulin; IL, interleukin; LABA, long-acting $\beta_{2}$ agonist; Q2W, every 2 weeks;
Q4W, every 4 weeks; SD, standard deviation; TSLP, thymic stromal lymphopoietin.

\section{Data Sharing Statement}

Data underlying the findings described in this paper may be obtained in accordance with AstraZeneca's data sharing policy described at https://astrazenecagrouptrials.phar macm.com/ST/Submission/Disclosure.

\section{Ethics Approval and Informed Consent}

This study was conducted in accordance with the ethical principles of the Declaration of Helsinki, International Conference for Harmonisation Good Clinical Practice guidelines, and applicable regulatory requirements. Approvals from Copernicus central Institutional Review Board (Durham, NC, USA) and local independent ethics committees were obtained, and all patients provided written informed consent in accordance with local requirements.

\section{Consent for Publication}

Not applicable. 


\section{Acknowledgments}

Medical writing support was provided by Richard Claes, $\mathrm{PhD}$, of PharmaGenesis London, London, UK, with funding from AstraZeneca and Amgen Inc, in accordance with Good Publication Practice 3 (GPP3) guidelines (http:// www.ismpp.org/gpp3).

\section{Author Contributions}

All authors made a significant contribution to the work reported, whether that was in the conception, study design, execution, acquisition of data, analysis and interpretation, or in all these areas; took part in drafting, revising or critically reviewing the article; gave final approval of the version to be published; have agreed on the journal to which the article was submitted; and agree to be accountable for all aspects of the work.

\section{Funding}

This study was co-sponsored by AstraZeneca and Amgen Inc. The sponsors were involved in the design of the study and analyses reported, and in the development of the manuscript.

\section{Disclosure}

JC received grants and personal fees from AstraZeneca during the conduct of the study, and has received grants and personal fees from Genentech, personal fees from Teva Pharmaceuticals, grants from Sanofi and personal fees from Vectura Group outside of the submitted work. MK, ÅH and $\mathrm{GC}$ are employees of AstraZeneca, and may own stocks in AstraZeneca. JRP is an employee of Amgen Inc and owns stock and stock options in Amgen Inc. In addition, JRP has patents: US20180296669A1 and WO2018191479A1, Treatment of Asthma with Anti-TSLP Antibody, pending. The authors report no other potential conflicts of interest for this work.

\section{References}

1. Global Asthma Network. Global asthma report 2018. Available from: http://www.globalasthmareport.org/. Accessed September 24, 2020.

2. Chung KF, Wenzel SE, Brozek JL, et al. International ERS/ATS guidelines on definition, evaluation and treatment of severe asthma. Eur Respir J. 2014;43(2):343-373. doi:10.1183/09031936.00202013

3. Global Initiative for Asthma. Global strategy for asthma management and prevention, updated 2019. Available from: https://ginasthma.org/ wp-content/uploads/2019/06/GINA-2019-main-report-June-2019wms.pdf. Accessed September 24, 2020.

4. Hekking PP, Wener RR, Amelink M, Zwinderman AH, Bouvy ML, Bel EH. The prevalence of severe refractory asthma. J Allergy Clin Immunol. 2015;135(4):896-902. doi:10.1016/j.jaci.2014.08.042
5. Peters SP, Ferguson G, Deniz Y, Reisner C. Uncontrolled asthma: a review of the prevalence, disease burden and options for treatment. Respir Med. 2006;100(7):1139-1151. doi:10.1016/j.rmed.2006.03.031

6. Chastek B, Korrer S, Nagar SP, et al. Economic burden of illness among patients with severe asthma in a managed care setting. J Manag Care Spec Pharm. 2016;22(7):848-861. doi:10.18553/jmcp.2016.22.7.848

7. Sadatsafavi M, Lynd L, Marra C, et al. Direct health care costs associated with asthma in British Columbia. Can Respir J. 2010;17 (2):74-80. doi:10.1155/2010/361071

8. Zeiger RS, Schatz M, Chen W, Li Q, Khatry DB, Tran TN. Adherent uncontrolled adult persistent asthma: characteristics and asthma outcomes. J Allergy Clin Immunol Pract. 2015;3(6):986-990. doi:10.1016/j.jaip.2015.06.016

9. Gerhardsson de Verdier M, Gustafson P, McCrae C, Edsbacker S, Johnston N. Seasonal and geographic variations in the incidence of asthma exacerbations in the United States. J Asthma. 2017;54 (8):818-824. doi:10.1080/02770903.2016.1277538

10. Szefler SJ, Raphiou I, Zeiger RS, Stempel D, Kral K, Pascoe S. Seasonal variation in asthma exacerbations in the AUSTRI and VESTRI studies. ERJ Open Res. 2019;5(2):00153-2018. doi:10.1183/23120541.00153-2018

11. DuBuske L, Newbold P, Wu Y, Trudo F. Seasonal variability of exacerbations of severe, uncontrolled eosinophilic asthma and clinical benefits of benralizumab. Allergy Asthma Proc. 2018;39 (5):345-349. doi:10.2500/aap.2018.39.4162

12. Staton TL, Arron JR, Olsson J, Holweg CTJ, Matthews JG, Choy DF. Seasonal variability of severe asthma exacerbations and clinical benefit from lebrikizumab. $J$ Allergy Clin Immunol. 2017;139 (5):1682-1684. doi:10.1016/j.jaci.2017.01.028

13. Johnston NW, Sears MR. Asthma exacerbations. 1: epidemiology. Thorax. 2006;61(8):722-728. doi:10.1136/thx.2005.045161

14. Galli SJ, Tsai M, Piliponsky AM. The development of allergic inflammation. Nature. 2008;454(7203):445-454. doi:10.1038/nature07204

15. Normansell R, Walker S, Milan SJ, Walters EH, Nair P. Omalizumab for asthma in adults and children. Cochrane Database Syst Rev. 2014; (1):CD003559. doi:10.1002/14651858.CD003559.pub4

16. Farne HA, Wilson A, Powell C, Bax L, Milan SJ. Anti-IL5 therapies for asthma. Cochrane Database Syst Rev. 2017;9:CD010834. doi:10.1002/14651858.CD010834.pub3

17. Zayed Y, Kheiri B, Banifadel M, et al. Dupilumab safety and efficacy in uncontrolled asthma: a systematic review and meta-analysis of randomized clinical trials. $J$ Asthma. 2018;1-10. doi:10.1080/ 02770903.2018.1520865

18. Busse WW, Morgan WJ, Gergen PJ, et al. Randomized trial of omalizumab (anti-IgE) for asthma in inner-city children. $N$ Engl $J$ Med. 2011;364(11):1005-1015. doi:10.1056/NEJMoa1009705

19. Ortega H, Chupp G, Bardin P, et al. The role of mepolizumab in atopic and nonatopic severe asthma with persistent eosinophilia. Eur Respir J. 2014;44(1):239-241. doi:10.1183/09031936.00220413

20. Gauvreau GM, O'Byrne PM, Boulet LP, et al. Effects of an anti-TSLP antibody on allergen-induced asthmatic responses. $N$ Engl $J$ Med. 2014;370(22):2102-2110. doi:10.1056/NEJMoa1402895

21. Corren J, Parnes J, Wang L, et al. Tezepelumab in adults with uncontrolled asthma. $N$ Engl J Med. 2017;377(10):936-946. doi:10.1056/NEJMoa1704064

22. Allakhverdi Z, Comeau MR, Jessup HK, et al. Thymic stromal lymphopoietin is released by human epithelial cells in response to microbes, trauma, or inflammation and potently activates mast cells. $J$ Exp Med. 2007;204(2):253-258. doi:10.1084/jem.20062211

23. Corren J, Ziegler SF. TSLP: from allergy to cancer. Nat Immunol. 2019;20(12):1603-1609. doi:10.1038/s41590-019-0524-9

24. Mitchell PD, O'Byrne PM. Epithelial-derived cytokines in asthma. Chest. 2017;151(6):1338-1344. doi:10.1016/j.chest.2016.10.042

25. World Health Organization. Review of the 2014-2015 influenza season in the northern hemisphere. Available from: https://www. who.int/wer/2015/wer9023/en/. Accessed September 24, 2020. 


\section{Publish your work in this journal}

The Journal of Asthma and Allergy is an international, peer-reviewed open-access journal publishing original research, reports, editorials and commentaries on the following topics: Asthma; Pulmonary physiology; Asthma related clinical health; Clinical immunology and the immunological basis of disease; Pharmacological interventions and new therapies. The manuscript management system is completely online and includes a very quick and fair peer-review system, which is all easy to use. Visit http://www.dovepress.com/testimonials.php to read real quotes from published authors. 